\title{
Cerebral Tissue Oxygenation Index Using Near-infrared Spectroscopy during Extracorporeal Cardio-pulmonary Resuscitation Predicted Good Neurological Recovery in a Patient with Acute Severe Anemia
}

\author{
Yasuaki Koyama, Taro Mizutani, Aiki Marushima, Aiko Sonobe, \\ Nobutake Shimojo and Satoru Kawano
}

\begin{abstract}
:
We herein report the clinical course of a patient who had a good neurological outcome despite severe anemia, high serum lactate levels, and a long period of time from cardiac arrest (CA) to extracorporeal cardiopulmonary resuscitation (ECPR) establishment. During the period of resuscitation, the tissue oxygenation index (TOI) values were measured continuously by a near-infrared spectroscopy monitoring device and were kept within the normal range. The TOI seems to reflect cerebral perfusion and the balance between the oxygen supply and demand in the brain during ECPR, thereby predicting the neurological outcome. Continuous TOI monitoring is useful for predicting the neurological outcome during ECPR.
\end{abstract}

Key words: ECPR, TOI, acute severe anemia, NIRO-Pulse, NIRS

(Intern Med 56: 2451-2453, 2017)

(DOI: 10.2169/internalmedicine.7826-16)

\section{Introduction}

Cardiac arrest (CA) decreases cerebral tissue oxygen and causes neurologic damage. The tissue oxygen decreases with severe anemia because the oxygen carrying capacity is reduced. The 2015 American Heart Association Guidelines stated that there is insufficient evidence to support the routine use of extracorporeal cardio-pulmonary resuscitation (ECPR) for patients with CA, but that in settings where it can be rapidly implemented, ECPR may be considered for select CA patients for whom the suspected etiology of the CA may be reversible (1). However, it is difficult to presume that $\mathrm{CA}$ has a reversible etiology and to anticipate a good neurological recovery during cardio-pulmonary resuscitation (CPR).

We herein report the interesting clinical course of a patient who had a good neurological outcome despite severe anemia, high serum lactate levels, and a long period of time (about one hour) from CA to ECPR establishment. During the period of resuscitation in the intensive-care unit (ICU), the tissue oxygenation index (TOI) values were measured continuously by a near-infrared spectroscopy (NIRS) monitoring device and were kept within the normal range in this patient. To our knowledge, this is the first report of continuous NIRS monitoring during ECPR in a patient with severe anemia.

\section{Case Report}

A 66-year-old woman with hypertrophic cardiomyopathy and chronic atrial fibrillation finished chemotherapy for diffuse large B-cell lymphoma. While eating breakfast on the day she was scheduled to be discharged, she developed ventricular fibrillation, and standard CPR was started immediately. Return of spontaneous circulation (ROSC) was not obtained despite a total of 18 defibrillation attempts and the administration of $11 \mathrm{mg}$ epinephrine and $450 \mathrm{mg}$ amiodarone. On arrival at our ICU, the TOI was above 50\% as measured by a NIRO-Pulse device (Hamamatsu Photonics, Hamamatsu, Japan; Fig. 1), which is an NIRS device that has been described elsewhere $(2,3)$. We decided to imple- 


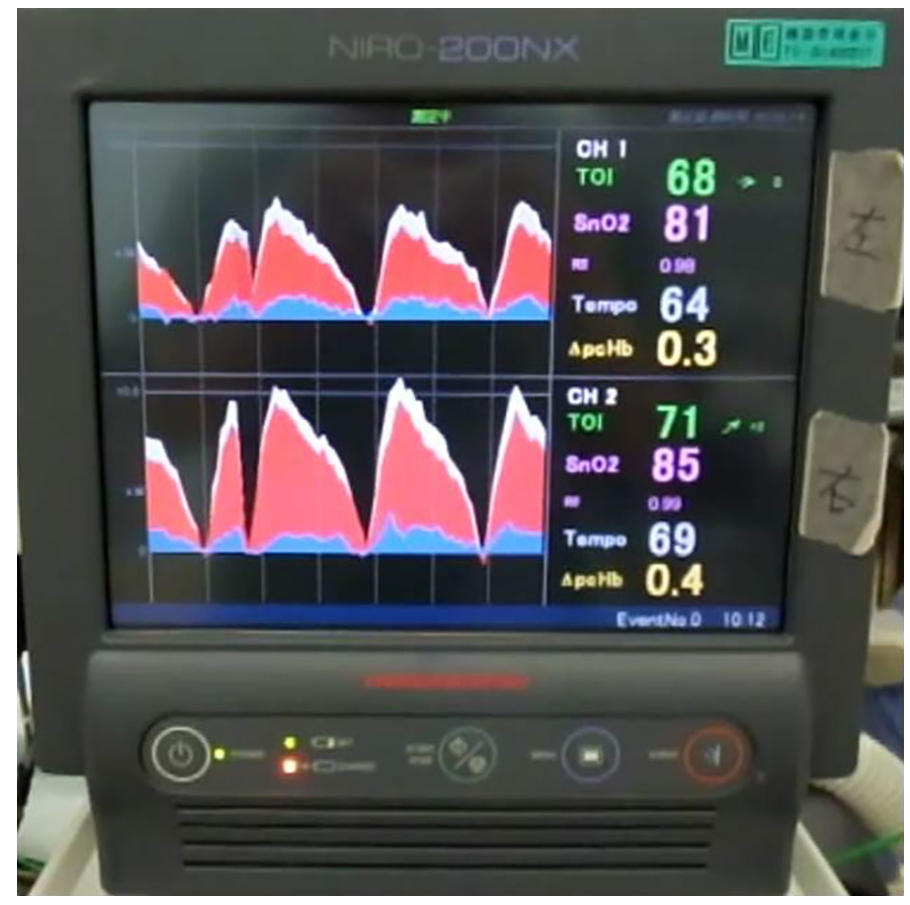

Figure 1. NIRO-Pulse display. The NIRO-Pulse is a nearinfrared spectroscopy (NIRS) device that can measure and display the following variables: TOI: tissue oxygenation index, $\mathrm{SnO}_{2}$ : saturation of the waveform, Tempo: pulse/compression rate, delta-pcHb: amplitude of delta-cHb. The white, red, and blue waveforms represent the delta-cHb (change in the total hemoglobin concentration), delta- $\mathrm{O}_{2} \mathrm{Hb}$ (change in the oxidized hemoglobin), and delta-HHb (change in the de-oxidized hemoglobin), respectively.

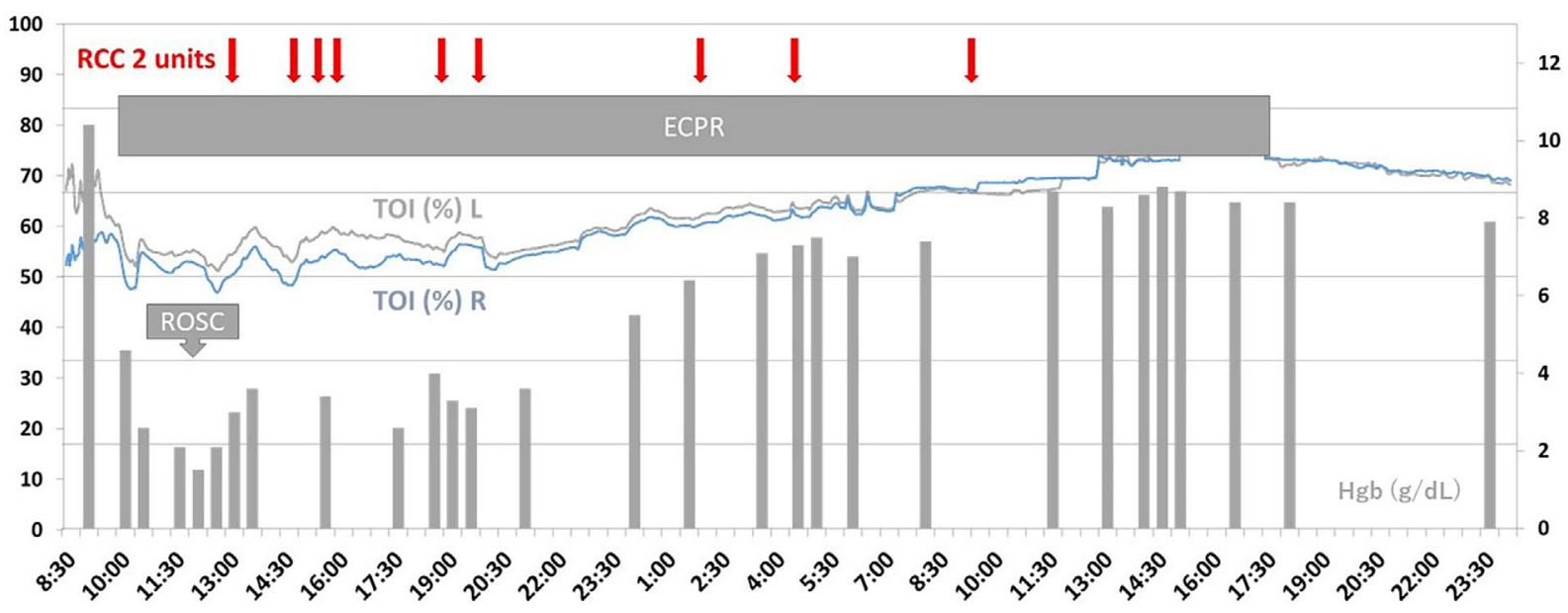

Figure 2. Changes in the TOI and hemoglobin values. The TOI $(\%) L$ and $R$ are measured on the left and right side of the patient's supraorbital region $2 \mathrm{~cm}$ above the eyebrows, respectively. Each arrow indicates (Japanese) 2 units of red cell concentrate (RCC) transfusion. ECPR: extracorporeal cardio-pulmonary resuscitation, TOI: tissue oxygenation index, ROSC: return of spontaneous circulation

ment ECPR. The CPR duration prior to the ICU arrival and that before ECPR was 45 and 58 minutes, respectively.

Her blood lactate level was $13.3 \mathrm{mmol} / \mathrm{L}$. Because the appropriate flow to establish ECPR was very difficult to ob- tain, a massive amount of crystalloids and colloids was given. Her hemoglobin level acutely decreased from $10.4 \mathrm{~g} /$ $\mathrm{dL}$ to $2.1 \mathrm{~g} / \mathrm{dL}$. Eventually, she obtained ROSC at 3 hours after CA. The TOI was largely maintained within the normal 
range of $50-60 \%$, and she underwent hypothermia therapy for approximately 30 hours (Fig. 2). She was successfully weaned off the ECPR device on day 2, and she was able to respond to verbal commands on day 3 . Her trachea was extubated on day 4, and her consciousness level was confirmed to be clear.

\section{Discussion}

Low hemoglobin or high serum lactate levels before ECPR and a prolonged interval from CA to ECPR predict poor neurological outcomes after successful ECPR (4). However, we implemented ECPR in this case because the initial TOI was within the normal range (3). An initial regional cerebral oxygen saturation $\geq 40 \%$ predicts a favorable outcome in patients with CA $(3,5,6)$. In our case, the TOI decreased just before ECPR implementation and increased to $55 \%$ immediately after that. It is likely that the oxygen carrying capacity could not be maintained by chest compression alone but was improved by ECPR.

The hemoglobin concentration decreased with large volumes of crystalloid and colloid infusion in this case. Although the scattering coefficient of tissue is known to depend on the wavelength, the scattering coefficient per se in TOI measurement is not affected by hemoglobin variation (7). Therefore, in this patient, the TOI values seemed to be measured accurately, and the TOI was maintained between $50 \%$ and $60 \%$, although it decreased temporarily when her hemoglobin concentration fell. Furthermore, the TOI increased gradually with an increase in the hemoglobin concentration by blood transfusion, probably because the oxygen carrying capacity improved. In addition, hypothermia therapy seemed to reduce the oxygen consumption, thereby maintaining the TOI values within the normal range.

Asphyxiated newborns who received hypothermia therapy and developed hypoxic-ischemic encephalopathy with a poor outcome showed a significantly higher TOI than normal (8). This higher TOI seemed to be caused by a decrease in the oxygen consumption of the injured neuronal cells and an increase in the oxygen carrying capacity due to ischemiareperfusion. Yagi et al. reported that a high initial TOI or continuously high TOI values during ECPR predict poor neurological outcomes (5). In the present case, the TOI values were not high but instead relatively low during the period after ECPR was discontinued, presumably because the oxygen consumption of the brain tissue was well-preserved. The effects of ROSC on the TOI were unremarkable, since the blood flow generated by the ECPR device was dominant in the initial phase.

Our present experience suggests that cerebral resuscitation can be successful if the TOI during CPR is maintained within the normal range, even in patients with prolonged CA, high serum lactate levels, and severe anemia. The TOI seems to reflect cerebral perfusion and the balance between the oxygen supply and demand in the brain during ECPR, thereby predicting the neurological outcome.

\section{Conclusion}

This is the first report regarding continuous NIRS monitoring during ECPR in a patient with severe anemia. The clinical course of this patient showed that continuous TOI monitoring was useful for predicting the neurological outcome during ECPR.

The authors state that they have no Conflict of Interest (COI).

\section{References}

1. Link MS, Berkow LC, Kudenchuk PJ, et al. Part 7: Adult advanced cardiovascular life support: 2015 American Heart Association Guidelines Update for Cardiopulmonary Resuscitation and Emergency Cardiovascular Care. Circulation 132: S444-S464, 2015.

2. Koyama Y, Wada T, Takamatsu Y, et al. Real-time cerebral hemoglobin concentration changes from NIRS; a valid evaluation method for quality CPR chest compression in out-of-hospital cardiac arrest. Circulation 128: A279, 2013.

3. Koyama Y, Wada T, Lohman BD, et al. A new method to detect cerebral blood flow waveform in synchrony with chest compression by near-infrared spectroscopy during CPR. Am J Emerg Med 1504-1508, 2013.

4. Ryu JA, Cho YH, Sung K, et al. Predictors of neurological outcomes after successful extracorporeal cardiopulmonary resuscitation. BMC Anesthesiol 15: 26, 2015.

5. Yagi T, Nagao K, Sakatani K, et al. Changes of cerebral oxygen metabolism and hemodynamics during ECPR with hypothermia measured by near-infrared spectroscopy: a pilot study. Adv Exp Med Biol 789: 121-128, 2013.

6. Nishiyama K, Ito N, Orita T, et al. Regional cerebral oxygen saturation monitoring for predicting interventional outcomes in patients following out-of-hospital cardiac arrest of presumed cardiac cause: a prospective, observational, multicentre study. Resuscitation 96: 135-141, 2015.

7. Yoshitani K, Kawaguchi M, Miura N, et al. Effects of hemoglobin concentration, skull thickness, and the area of the cerebrospinal fluid layer on near-infrared spectroscopy measurements. Anesthesiology 106: 458-462, 2007.

8. Ancora G, Maranella E, Grandi S, et al. Early predictors of short term neurodevelopmental outcome in asphyxiated cooled infants. A combined brain amplitude integrated electroencephalography and near infrared spectroscopy study. Brain Dev 35: 26-31, 2013.

The Internal Medicine is an Open Access article distributed under the Creative Commons Attribution-NonCommercial-NoDerivatives 4.0 International License. To view the details of this license, please visit (https://creativecommons.org/licenses/ by-nc-nd/4.0/).

(C) 2017 The Japanese Society of Internal Medicine Intern Med 56: 2451-2453, 2017 